\title{
Completely $q$-multiplicative functions: the Mellin transform approach
}

by

\section{Peter J. Grabner (Graz)}

1. Introduction. In [Ge68] and [De72] A. O. Gelfond and H. Delange investigate properties of arithmetic functions which satisfy functional equations closely related to the $q$-ary digit expansion of the integers:

$$
\begin{aligned}
& f\left(a q^{r}+b\right)=f\left(a q^{r}\right)+f(b) \quad \text { or } \\
& f\left(a q^{r}+b\right)=f\left(a q^{r}\right) f(b)
\end{aligned}
$$

for all $r \geq 0, a \geq 0$ and $0 \leq b<q^{r}$. Note that these equations force $f(0)=0$ or $f(0)=1$ respectively. These functions are called $q$-additive and $q$-multiplicative, respectively. It is easy to see that the functional equations imply that these functions are defined for all integers, when the values $f\left(a q^{r}\right)$ are known for $1 \leq a \leq q-1$ and all $r \geq 0$.

In [MM83] the case of $q$-additive functions is studied with the help of the Dirichlet generating function of $f(n)$ under the additional assumption that $\sum_{k=0}^{\infty} f\left(a q^{k}\right) z^{k}$ is rational. The "typical" result for the summatory function $\sum_{n<N} f(n)$ is a (finite) linear combination of terms $N^{\alpha}(\log N)^{k} \varphi\left(\log _{q} N\right)$, where $k \geq 0$ is an integer and $\varphi$ is a continuous periodic function of period 1. A well known example in this context is the binary sum-ofdigits function $s(n)$ for which Delange's summation formula (cf. [De75]) holds:

$$
\sum_{n<N} s(n)=\frac{N}{2} \log _{2} N+N \varphi\left(\log _{2} N\right) .
$$

Here the approach via Dirichlet generating functions gives a new and comfortable proof for this result and also allows the computation of the Fourier coefficients of $\varphi$. In [FGKPT92] Mellin-Perron's summation formula

The author is supported by the Austrian Science Foundation (Project Nr. P-8274PHY). 
(cf. $[$ Ap84])

$$
\sum_{n<N} a_{n}\left(1-\frac{n}{N}\right)=\frac{1}{2 \pi i} \int_{c-i \infty}^{c+i \infty} \sum_{n=1}^{\infty} \frac{a_{n}}{n^{s}} \frac{N^{s}}{s(s+1)} d s
$$

is used to find asymptotic expansions for digital sums, e.g. $\sum_{n<N} 2^{s(n)}$. In a series of papers (cf. [Al87] for a detailed survey) the notion of $q$-automatic sequences is investigated; there are some connections to the sequences discussed here (see the beginning of Section 2).

In this paper we want to investigate asymptotic properties of the summatory functions $\sum_{n<N} f(n)$ of completely $q$-multiplicative functions (cf. Section 2) by the use of the Mellin transform and the summation formula (1.2). For this purpose we have to consider the analytical behaviour of the Dirichlet generating functions of $q$-multiplicative functions. Note that some special Dirichlet series and infinite products connected with $q$-ary digit counting functions are considered in [AC85].

In a final section of the present paper the summatory function of $(-1)^{s(p n)}$ ( $p$ prime) is analysed as example for the technique developed in Section 2. The main term of the asymptotics can be extracted from [Du83]. For a full asymptotic expansion see also [GKS92]. Finally, an application to the computation of the Hausdorff measure of a subset of the Sierpiński gasket is demonstrated.

2. Completely $q$-multiplicative functions. As Delange's results show that the behaviour of the summatory function can be very irregular in a general situation, we restrict our investigations to functions which satisfy

$$
f\left(\sum_{l=0}^{L} \varepsilon_{l} q^{l}\right)=\prod_{l=0}^{L} f\left(\varepsilon_{l}\right)
$$

and call these functions completely q-multiplicative. Special cases of these functions are exponential functions of the sum-of-digits function, which were studied in e.g. [Ha77], [St89] and [FGKPT92]. Notice that there are qmultiplicative functions which are $q$-automatic (cf. [Al87]), but there are also $q$-multiplicative functions which are not $q$-automatic, and vice versa; e.g. $2^{s(n)}$, where $s(n)$ is the binary sum-of-digits function, is 2-multiplicative, but not 2-automatic; the Thue-Morse sequence $(-1)^{s(n)}$ is 2-multiplicative and 2-automatic, and the Rudin-Shapiro sequence is 2-automatic but not 2-multiplicative.

Our first objective is to find an analytic continuation of the Dirichlet generating function of $f(n)$

$$
\gamma(s)=\sum_{n=1}^{\infty} \frac{f(n)}{n^{s}}
$$


The following proposition generalizes a result in [AC85]; there is a similar theorem for the Dirichlet generating functions of $q$-automatic sequences (cf. [Al87]).

Proposition 1. $\gamma(s)$ has an analytic continuation to the whole complex plane.

(1) If $\alpha_{f}=\sum_{n<q} f(n) \neq 0$ and $\log _{q} \alpha_{f} \notin \mathbb{Z}, \gamma(s)$ is meromorphic with (possible) simple poles at $\log _{q} \alpha_{f}-n+2 k \pi i /(\log q)$, where $n \in \mathbb{N}_{0}$ and $k \in \mathbb{Z}$. Moreover, $\gamma(0)=-1$ and $\gamma(-n)=0$ for $n \in \mathbb{N}$.

(2) If $\alpha_{f} \neq 0$ and $\log _{q} \alpha_{f} \in \mathbb{Z}$ there are poles at $\log _{q} \alpha_{f}-n+2 k \pi i /(\log q)$ for $\log _{q} \alpha_{f} \leq n$ and $k \in \mathbb{Z} \backslash\{0\}$. If $\alpha_{f}>1$ there are additional poles at $0<n \leq \log _{q} \alpha_{f}$. If $\alpha_{f}<1$ there are zeros at $\log _{q} \alpha_{f} \leq n<0$.

(3) If $\alpha_{f}=0$ then $\gamma(s)$ is an entire function with $\gamma(0)=-1$ and $\gamma(-k)=$ 0 for $k \in \mathbb{N}$.

Proof. We first note that

$$
G(z)=\sum_{n=0}^{\infty} f(n) z^{n}=\prod_{k=0}^{\infty}\left(1+f(1) z^{q^{k}}+\ldots+f(q-1) z^{(q-1) q^{k}}\right) .
$$

From this equality we derive

$$
\Gamma(s) \gamma(s)=\int_{0}^{\infty}\left(G\left(e^{-x}\right)-1\right) x^{s-1} d x \quad \text { for } \operatorname{Re} s>\sigma_{0}
$$

for a suitable $\sigma_{0}$ and therefore we can find all poles of $\Gamma(s) \gamma(s)$ by investigating the asymptotic properties of $G\left(e^{-t}\right)$ as $t \rightarrow 0$ and $t \rightarrow \infty$ (cf. [FRS85]).

In order to apply Mellin's inversion technique we investigate the function

$$
\delta(s)=\int_{0}^{\infty} \log \left(1+f(1) e^{-x}+\ldots+f(q-1) e^{-(q-1) x}\right) x^{s-1} d x
$$

(if there is a zero of the term under the logarithm on the real axis, we rotate the line of integration in this integral to $\arg x=\varepsilon)$. Clearly $\log (1+$ $\left.f(1) e^{-x}+\ldots+f(q-1) e^{-(q-1) x}\right)=O\left(e^{-x}\right)$ as $x \rightarrow \infty$, thus the poles of the function (2.2) originate from the behaviour of the logarithm as $x \rightarrow 0$. We note that $\delta(\sigma+i t)=O\left(t^{-M}\right)$ for all $M>0$, because the integrand is infinitely differentiable. We now have to distinguish two cases:

(a) $\alpha_{f}=1+f(1)+\ldots+f(q-1) \neq 0$. In this case there is a Taylor expansion

$$
\log \left(1+f(1) e^{-x}+\ldots+f(q-1) e^{-(q-1) x}\right)=\log \alpha_{f}+\sum_{k=1}^{\infty} a_{k} x^{k} .
$$

Thus by the basic properties of the Mellin transform $\delta(s)$ has a meromorphic continuation to the whole complex plane with poles at $s=0,-1,-2, \ldots$ and residues $\log \alpha_{f}, a_{1}, a_{2}, \ldots$ 
Therefore we derive

$$
\int_{0}^{\infty} \log G\left(e^{-x}\right) x^{s-1} d x=\frac{\delta(s)}{1-q^{-s}}
$$

for $\operatorname{Re} s>\sigma_{0}=0$. By Mellin's inversion formula we obtain

$$
\log G\left(e^{-x}\right)=\frac{1}{2 \pi i} \int_{1-i \infty}^{1+i \infty} \frac{\delta(s)}{1-q^{-s}} x^{-s} d s
$$

and shifting the line of integration to the left and taking residues into account yields

$$
\log G\left(e^{-x}\right)=-\log _{q} \alpha_{f} \log x+\psi_{0}+\sum_{k \in \mathbb{Z} \backslash\{0\}} \psi_{k} x^{\vartheta_{k}}+\sum_{l=1}^{\infty} d_{l} x^{l},
$$

as an asymptotic expansion, where the coefficients $\psi_{k}$ and $d_{l}$ can be expressed by values of $\delta(s)$ and $\vartheta_{k}=2 k \pi i /(\log q)$.

Taking the exponential of $(2.5)$ yields

$$
G\left(e^{-x}\right)=x^{-\log _{q} \alpha_{f}} \Phi\left(\log _{q} x\right) \sum_{k=0}^{\infty} g_{k} x^{k}, \quad g_{0} \neq 0,
$$

where $\Phi$ is a continuous periodic function of period 1 . Therefore we conclude that $\Gamma(s) \gamma(s)$ has an analytic continuation to the whole complex plane with (possible) simple poles at $s=\log _{q} \alpha_{f}-n+\vartheta_{k}$, where $n=0,1, \ldots$ and $k \in \mathbb{Z}$. From the subtraction of 1 in (2.2) there originates a simple pole with residue -1 at $s=0$, at least in the case $\log _{q} \alpha_{f} \notin \mathbb{Z}$.

(b) $\alpha_{f}=0$. In this case we have, for some $k>0$,

$$
1+f(1) e^{-x}+\ldots+f(q-1) e^{-(q-1) x}=c_{k} x^{k}+c_{k+1} x^{k+1}+\ldots
$$

with $c_{k} \neq 0$ and

$$
\log \left(1+f(1) e^{-x}+\ldots+f(q-1) e^{-(q-1) x}\right)=k \log x+\log c_{k}+\sum_{l=1}^{\infty} a_{l} x^{l} .
$$

Again by the basic properties of the Mellin transform, $\delta(s)$ has a double pole at $s=0$ with principal part $-k / s^{2}+\left(\log c_{k}\right) / s$ and simple poles at $s=-1,-2, \ldots$ with residues $a_{1}, a_{2}, \ldots$

As in case (a), by using Mellin's inversion formula, shifting the line of integration to the left and taking residues into account (note the triple pole at $s=0$ ), we obtain

$$
\log G\left(e^{-x}\right)=-\frac{k}{\log q} \log ^{2} x+O(\log x) .
$$


The full asymptotic expansion could be found as above, but it is not needed in the following. Exponentiating (2.7) yields

$$
G\left(e^{-x}\right)=O\left(\exp \left(-\frac{k}{\log q} \log ^{2} x\right) x^{\beta}\right)
$$

for some $\beta$.

Again we find the analytic continuation of $\Gamma(s) \gamma(s)$ by the basic properties of the Mellin transform. The only pole of this function at $s=0$ with residue -1 originates from the term -1 in (2.2). Dividing by $\Gamma(s)$ yields the desired conclusion.

We are now ready to formulate the

THEOREM. Let $f$ be a completely q-multiplicative function satisfying

$$
|1+f(1)+f(2)+\ldots+f(q-1)|>\max _{0 \leq a<q}|f(a)| .
$$

Then

$$
F(N)=\sum_{n<N} f(n)=N^{\varrho} e^{i \alpha \log _{q} N} \psi\left(\log _{q} N\right),
$$

where $\psi$ is a continuous, periodic function of period $1, \varrho=\log _{q}|F(q)|$ and $\alpha=\arg F(q)$. The Fourier expansion of $\psi$ is given by

$$
\begin{aligned}
\widehat{\psi}(k)= & \frac{1}{\Gamma\left(\varrho+1+\chi_{k}\right) \log q} \int_{0}^{\infty}\left(f(1) e^{-x}+\ldots+f(q-1) e^{-(q-1) x}\right. \\
& +\left(1+f(1) e^{-x}+\ldots+f(q-1) e^{-(q-1) x}-F(q)\right) \\
& \left.\times\left(G\left(e^{-q x}\right)-1\right)\right) x^{\varrho+\chi_{k}-1} d x,
\end{aligned}
$$

where $\chi_{k}=(2 k \pi i+\alpha) / \log q$. In the case that $F(q)$ is real and positive $\widehat{\psi}(0)$ can be interpreted as the mean value of $F(N) / N^{\varrho}$.

Proof. We split the proof into two parts: first we show (2.9) and then we use our information on the Dirichlet series to give the formula for the Fourier coefficients. Let $N=\sum_{l=0}^{L} \varepsilon_{l} q^{l}$ and $N_{p}=\sum_{l=p}^{L} \varepsilon_{l} q^{l}$. Then we have

$$
\begin{aligned}
F(N) & =\sum_{n<N} f(n)=\sum_{n<N_{L}} f(n)+\sum_{p=0}^{L-1} \sum_{n=N_{p+1}}^{N_{p}-1} f(n) \\
& =F\left(\varepsilon_{L} q^{L}\right)+\sum_{p=0}^{L-1} f\left(N_{p+1}\right) F\left(\varepsilon_{p} q^{p}\right) .
\end{aligned}
$$

Thus we have reduced the problem to the computation of $F\left(\varepsilon q^{k}\right)$. Now,

$$
F\left(\varepsilon q^{k}\right)=\sum_{a<\varepsilon} f(a) F\left(q^{k}\right)=F(\varepsilon) F(q)^{k} .
$$


Therefore we have

$$
F(N)=\sum_{p=0}^{L} \prod_{k=p+1}^{L} f\left(\varepsilon_{k}\right) F\left(\varepsilon_{p}\right) F(q)^{p} .
$$

We now extract the main term

$$
F(N)=F(q)^{L} \sum_{p=0}^{L} \prod_{k=p+1}^{L} f\left(\varepsilon_{k}\right) F\left(\varepsilon_{p}\right) F(q)^{p-L}
$$

and set

$$
\varphi\left(\sum_{l=0}^{\infty} \varepsilon_{l} q^{-l}\right)=\sum_{l=0}^{\infty} \prod_{k=0}^{l-1} f\left(\varepsilon_{k}\right) F\left(\varepsilon_{l}\right) F(q)^{-l} .
$$

Using (2.8) we deduce that this series is convergent (this shows that the assumption on $f$ is even necessary for the behaviour of the summatory function as indicated in the theorem). We now have to show that $\varphi$ is well-defined and continuous. Continuity at all points which are not $q$-adic rationals is a consequence of the rapid convergence of the geometric series. It remains to analyze the $q$-adic rationals: let

$$
x=\sum_{l=0}^{L} \varepsilon_{l} q^{-l}=\sum_{l=0}^{L-1} \varepsilon_{l} q^{-l}+\left(\varepsilon_{L}-1\right) q^{-L}+\sum_{l=L+1}^{\infty}(q-1) q^{-l} ;
$$

then we have to show that the value of $\varphi(x)$ is the same for both representations of $x$. We have to prove

$$
\begin{aligned}
\varphi(x)= & \sum_{l=0}^{L} \prod_{k=0}^{l-1} f\left(\varepsilon_{k}\right) F\left(\varepsilon_{l}\right) F(q)^{-l} \\
= & \sum_{l=0}^{L-1} \prod_{k=0}^{l-1} f\left(\varepsilon_{k}\right) F\left(\varepsilon_{l}\right) F(q)^{-l}+\prod_{k=0}^{L-1} f\left(\varepsilon_{k}\right) F\left(\varepsilon_{L}-1\right) F(q)^{-L} \\
& +\prod_{k=0}^{L-1} f\left(\varepsilon_{k}\right) F\left(\varepsilon_{L}-1\right) \sum_{l=L+1}^{\infty} f(q-1)^{l-L-1} F(q-1) F(q)^{-l} .
\end{aligned}
$$

Summing up the infinite sum in the last line and collecting the result and the other terms yields this equality. Thus $\varphi$ is continuous and well-defined. We note that $\varphi(1)=1$ and $\varphi(q)=F(q)$.

We now derive

$$
\begin{aligned}
F(N) & =F(q)^{\left[\log _{q} N\right]} \varphi\left(\frac{N}{q^{\left[\log _{q} N\right]}}\right) \\
& =N^{\varrho} e^{i \alpha \log _{q} N} F(q)^{-\left\{\log _{q} N\right\}} \varphi\left(q^{\left\{\log _{q} N\right\}}\right),
\end{aligned}
$$


where $[x]$ and $\{x\}$ denote the integer and the fractional part of $x$ as usual. Setting $\psi\left(\log _{q} N\right)=F(q)^{-\left\{\log _{q} N\right\}} \varphi\left(q^{\left\{\log _{q} N\right\}}\right)$ yields the first part of the theorem.

For the computation of the Fourier coefficients we again use the information on the function $G\left(e^{-t}\right)(2.1)$ that we gathered in the proof of Proposition 1. Using the functional equation (notice that this is a functional equation "à la Mahler" [Ma29])

$$
G\left(e^{-x}\right)=\left(1+f(1) e^{-x}+\ldots+f(q-1) e^{-(q-1) x}\right) G\left(e^{-q x}\right)
$$

we derive

$$
\begin{aligned}
& G\left(e^{-x}\right)-1 \\
& =F(q)\left(G\left(e^{-q x}\right)-1\right)+f(1) e^{-x}+\ldots+f(q-1) e^{-(q-1) x} \\
& \quad+\left(1+f(1) e^{-x}+\ldots+f(q-1) e^{-(q-1) x}-F(q)\right)\left(G\left(e^{-q x}\right)-1\right) .
\end{aligned}
$$

Taking the Mellin transform of this equation and dividing by $\Gamma(s)$ yields

$$
\begin{aligned}
\eta(s):= & \gamma(s)\left(1-F(q) q^{-s}\right) \\
= & f(1)+\frac{f(2)}{2^{s}}+\ldots+\frac{f(q-1)}{(q-1)^{s}} \\
& +\sum_{n=1}^{\infty}\left(\frac{f(q n+1)}{(q n+1)^{s}}+\ldots+\frac{f(q n+q-1)}{(q n+q-1)^{s}}\right. \\
& \left.-\frac{f(q n+1)+\ldots+f(q n+q-1)}{(q n)^{s}}\right) .
\end{aligned}
$$

By the formula for the abscissa of convergence (cf. [HR15]) this last series is convergent for $\sigma>\log _{q} \max _{0 \leq a<q}|f(a)|=: \sigma_{0}$. Note that this abscissa of convergence is left of $\varrho$. On the other hand, $\eta(s)$ is absolutely convergent, e.g. in the half plane $\sigma>\varrho+1$, where we have $\eta(\sigma+i t)=O(1)$ as $t \rightarrow \infty$. For every $\varepsilon>0$ we have $\eta\left(\sigma_{0}+\varepsilon+i t\right)=o(t)$ (cf. [HR15]). Thus by Lindelöf's theorem we have $\eta(\varrho+i t)=O\left(t^{\alpha}\right)$ for some $\alpha<1$.

By Mellin-Perron's summation formula we have

$$
\begin{aligned}
& \sum_{n<N} F(n) \\
= & \sum_{n<N}(N-n) f(n)=N+\frac{N}{2 \pi i} \int_{\varrho+1-i \infty}^{\varrho+1+i \infty} \frac{\eta(s)}{1-F(q) q^{-s}} \frac{N^{s}}{s(s+1)} d s .
\end{aligned}
$$

We shift the line of integration to $\sigma=\varrho-\varepsilon$ and compute the residues at the zeros of $1-F(q) q^{-s}$ : 


$$
\begin{aligned}
\operatorname{Res}_{s=\varrho+\chi_{k}} \frac{\eta(s) N^{s}}{\left(1-F(q) q^{-s}\right) s(s+1)} \\
=\frac{\eta\left(\varrho+\chi_{k}\right)}{\left(\varrho+\chi_{k}\right)\left(\varrho+\chi_{k}+1\right) \log q} e^{2 k \pi i \log _{q} N} e^{i \alpha \log _{q} N} N^{\varrho} .
\end{aligned}
$$

This yields the asymptotic expansion

$$
\sum_{n<N} F(n)=N^{\varrho+1} e^{i \alpha \log _{q} N} \Psi\left(\log _{q} N\right)+O\left(N^{\varrho+1-\varepsilon}\right),
$$

where the Fourier coefficients of $\Psi$ come from the values of the residues:

$$
\widehat{\Psi}(k)=\frac{\eta\left(\varrho+\chi_{k}\right)}{\left(\varrho+\chi_{k}\right)\left(\varrho+\chi_{k}+1\right) \log q} .
$$

By a pseudo-Tauberian argument used in [FGKPT92] it is possible to derive the Fourier coefficients of $\psi$ from the Fourier expansion of $\Psi$.

Proposition 2. Let $h$ be a continuous function, periodic with period 1 , and let $\tau$ be a complex number with $\operatorname{Re} \tau>0$. Then there exists a continuously differentiable function $H$ of period 1 such that

$$
\begin{aligned}
\sum_{n<N} n^{\tau} h\left(\log _{q} n\right) & =N^{\tau+1} H\left(\log _{q} N\right)+o\left(N^{\tau+1}\right), \\
\int_{0}^{1} H(x) d x & =\frac{1}{\tau+1} \int_{0}^{1} h(x) d x .
\end{aligned}
$$

The proof of this proposition is a rephrasing of the proof of [FGKPT92, Proposition 2].

Using Proposition 2 with $n^{\tau} h\left(\log _{q} n\right)=F(n)$ and $\tau=\varrho+\chi_{k}$ yields

$$
\widehat{\psi}(k)=\frac{\eta\left(\varrho+\chi_{k}\right)}{\left(\varrho+\chi_{k}\right) \log q} .
$$

Computing the Mellin transform of (2.15) yields the integral representation of $\eta(s)$ and the theorem is proved.

3. Examples and applications. As an example for completely $q$ multiplicative functions we consider

$$
\sum_{n<N}(-1)^{s(p n)}
$$

where $s(n)$ is the dyadic sum-of-digits function and $p$ is a prime which has 2 as a primitive root (the method discussed above can also be applied to other cases, but then some parts of the computation become much more complicated). The case $p=3$ was considered in [Ne69] and [Co83], where it 
is proved that

$$
\sum_{n<N}(-1)^{s(3 n)}=N^{\log _{4} 3} F\left(\log _{4} N\right)+\frac{\eta(N)}{3},
$$

where

$$
\eta(N)= \begin{cases}0 & \text { if } N \text { is even, } \\ (-1)^{s(3 N-1)} & \text { if } N \text { is odd }\end{cases}
$$

and the continuous function $F$ is strictly positive and nowhere differentiable. This result shows that the sum-of-digits function of the multiples of 3 attains even values more often than odd ones. In [GKS92] the sum (3.1) is investigated in general and the asymptotic expansion is computed by investigating the eigenvalues of certain matrices. It seems to be difficult to compute the extremal values of the fractal function (or even to find out whether it takes only positive values or not) in the general situation. In a forthcoming paper [Gr93] the author proves that for $p=5$ the fractal function is always positive and computes its extremal values and the mean value.

Let $\xi$ be a $p$ th root of unity in the sequel; then the function

$$
f_{\xi}(n)=(-1)^{s(n)} \xi^{n}
$$

is completely $2^{p-1}$-multiplicative. The theorem shows that the value of $F_{\xi}\left(2^{p-1}\right)$ determines the asymptotic behaviour of $F(N)$. In this case we have

$$
F_{\xi}\left(2^{p-1}\right)=\prod_{k=0}^{p-2}\left(1-\xi^{2^{k}}\right)=p .
$$

(Here we have used the assumption that 2 is a primitive root $\bmod p$. For general $p$ it is much more difficult to compute this product (cf. [GKS92]).) Thus we have

$$
\begin{aligned}
\sum_{n<N}(-1)^{s(p n)} & =\frac{1}{p}\left(\sum_{n<p N}(-1)^{s(n)}+\sum_{\xi} \sum_{n<p N} f_{\xi}(n)\right) \\
& =N^{\frac{1}{p-1} \log _{2} p} \psi_{p}\left(\frac{1}{p-1} \log _{2} N\right)+\frac{\eta_{p}(N)}{p},
\end{aligned}
$$

where

$$
\eta_{p}(N)= \begin{cases}0 & \text { if } N \text { is even } \\ (-1)^{s(p N-1)} & \text { if } N \text { is odd }\end{cases}
$$

For $p=3$ the function $\psi_{3}$ satisfies

$$
1.154700538 \ldots=\frac{2}{\sqrt{3}} \leq \psi_{3}(x) \leq \frac{55}{3}\left(\frac{3}{65}\right)^{\log _{4} 3}=1.601958421 \ldots
$$


(cf. [Co83]) and the mean value is $1.4092203477 \ldots$ (cf. [FGKPT92]). For $p=5$ the extremal values are

$$
\begin{aligned}
0.8380851409 \ldots=\frac{7}{10}\left(\frac{15}{11}\right)^{\log _{16} 5} & \leq \psi_{5}(x) \\
& \leq \frac{9}{10}\left(\frac{60}{13}\right)^{\log _{16} 5}=2.186770740 \ldots
\end{aligned}
$$

and the mean value is $1.56205765115 \ldots$ (cf. [Gr93]).

Let us now consider the Sierpiński gasket.

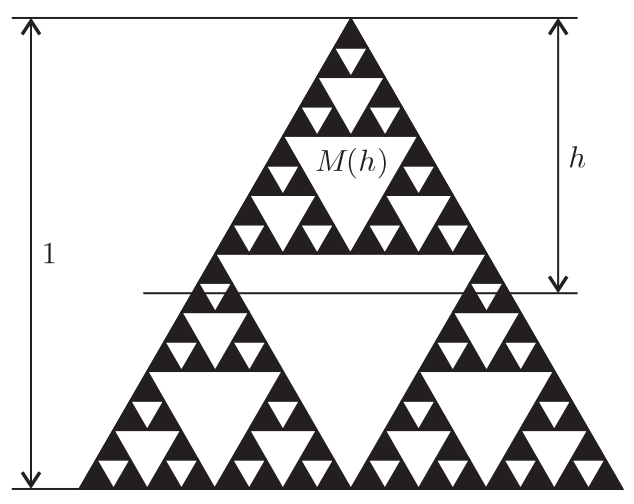

Fig. 1

We want to compute the Hausdorff measure $\mathcal{H}$ of dimension $\log _{2} 3$ of the subset $M(h)$ of the gasket obtained by cutting the gasket along a horizontal line as in Figure 1 (the measure is normed, such that $\mathcal{H}(G)=1$ ). Let $h$ be a dyadic rational

$$
h=\sum_{l=1}^{L} \frac{\varepsilon_{l}}{2^{l}} .
$$

Then the set $M(h)$ consists of

$$
\sum_{n<2^{L} h} 2^{s(n)}
$$

triangles of measure $3^{-L}$. This follows immediately from the fact that the positions of odd entries in Pascal's triangle up to a given power of 2 form a finite approximation to the Sierpiński gasket. The number of odd entries in the $n$th line of Pascal's triangle is given by $2^{s(n)}$ (cf. [Ha77]). We now have

$$
\mathcal{H}(M(h))=\sum_{n<2^{L} h} 2^{s(n)} 3^{-L}=\sum_{l=1}^{L} \varepsilon_{l} 2^{\varepsilon_{1}+\ldots+\varepsilon_{l-1}} 3^{-l} .
$$


By approximating an arbitrary real number $h$ by dyadic rationals and observing that $\mathcal{H}(M(h))$ is a continuous function of $h$, we obtain

$$
\mathcal{H}(M(h))=\sum_{l=1}^{\infty} \varepsilon_{l} 2^{\varepsilon_{1}+\ldots+\varepsilon_{l-1}} 3^{-l}=h^{\log _{2} 3} F\left(\log _{2} h\right),
$$

where $F$ is a continuous periodic function of period 1, which also occurred in [FGKPT92] when investigating the summatory function (3.3). The periodicity of $F$ reflects the self-similarity of the gasket.

Acknowledgements. I am indebted to an anonymous referee for valuable remarks concerning the presentation of the paper and for pointing out the references [MM83] and [GKS92].

\section{References}

[Al87] J.-P. Allouche, Automates finis en théorie des nombres, Exposition. Math. 5 (1987), 239-266.

[AC85] J.-P. Allouche and H. Cohen, Dirichlet series and curious infinite products, Bull. London Math. Soc. 17 (1985), 531-538.

[AS88] J.-P. Allouche and J. O. Shallit, Sums of digits and the Hurwitz zeta function, in: Analytic Number Theory, Lecture Notes in Math. 1434, Springer, Berlin, 1988, 19-30.

[Ap84] T. M. A postol, Introduction to Analytic Number Theory, Springer, Berlin, 1984.

[Co83] J. Coquet, A summation formula related to the binary digits, Invent. Math. 73 (1983), 107-115.

[De72] H. Delange, Sur les fonctions q-additives ou q-multiplicatives, Acta Arith. 21 (1972), 285-298.

[De75] —, Sur la fonction sommatoire de la fonction "Somme des Chiffres", Enseign. Math. (2) 21 (1975), 31-47.

[Du83] J.-M. Dumont, Discrépance des progressions arithmétiques dans la suite de Morse, C. R. Acad. Sci. Paris 297 (1983), 145-148.

[FGKPT92] P. Flajolet, P. J. Grabner, P. Kirschenhofer, H. Prodinger and R. F. Tichy, Mellin transforms and asymptotics: digital sums, Theoret. Comput. Sci. (to appear).

[FRS85] P. Flajolet, M. Regnier and R. Sedgewick, Some uses of the Mellin integral transform in the analysis of algorithms, in: Combinatorial Algorithms on Words, A. Apostolico and Z. Galil (eds.), Springer, Berlin, 1985, $241-254$.

[Ge68] A. O. Gelfond, Sur les nombres qui ont des propriétés additives et multiplicatives données, Acta Arith. 13 (1968), 259-266.

[GKS92] S. Goldstein, K. A. Kelly and E. R. Speer, The fractal structure of rarefied sums of the Thue-Morse sequence, J. Number Theory 42 (1992), $1-19$.

[Gr93] P. J. Grabner, A note on the parity of the sum-of-digits function, manuscript. 
[Ha77] H. Harborth, Number of odd binomial coefficients, Proc. Amer. Math. Soc. 62 (1977), 19-22.

[HR15] G. H. Hardy and M. Riesz, The General Theory of Dirichlet's Series, Cambridge University Press, 1915.

[Ma29] K. Mahler, Arithmetische Eigenschaften der Lösungen einer Klasse von Funktionalgleichungen, Math. Ann. 101 (1929), 342-366.

[MM83] J.-L. Mauclaire and L. Murata, On q-additive functions, II, Proc. Japan Acad. 59 (1983), 441-444.

[Ne69] D. J. New man, On the number of binary digits in a multiple of three, Bull. Amer. Math. Soc. 21 (1969), 719-721.

[St89] A. H. Stein, Exponential sums of digit counting functions, in: Théorie des nombres, Comptes Rendus de la Conférence Internationale de Théorie des Nombres tenue à l'Université Laval en 1987, J. M. De Koninck and C. Levesque (eds.), W. de Gruyter, Berlin, 1989, 861-868.

[Sto77] K. B. Stolarsky, Power and exponential sums of digital sums related to binomial coefficient parity, SIAM J. Appl. Math. 32 (1977), 717-730.

INSTITUT FÜR MATHEMATIK

TECHNISCHE UNIVERSITÄT GRAZ

STEYRERGASSE 30

8010 GRAZ, AUSTRIA

E-mail: GRABNER@FTUG.DNET.TU-GRAZ.AC.AT

Received on 14.7.1992

and in revised form on 22.3.1993 